\title{
Conceptual processing of text during skimming and rapid sequential reading
}

\author{
MICHAEL E. J. MASSON \\ University of Victoria, Victoria, British Columbia V8W 2Y2, Canada
}

\begin{abstract}
Potential conceptual processing difficulties associated with abbreviating the duration of eye fixations during reading were explored using a rapid sequential visual presentation (RSVP) paradigm in which each word of a text was briefly presented (for as little as $86 \mathrm{msec}$ ) at a fixed location. Question answering and accuracy of summaries associated with RSVP were found to be inferior to performance obtained when subjects skimmed conventionally presented passages. RSVP performance was enhanced by inserting brief pauses between sentences. The pauses produced only minor changes in the perceptual characteristics of RSVP, but they probably allowed subjects to more readily process information that had been buffered in working memory. These results imply that eye fixation durations in conventional reading reflect the time constraints of conceptual processing operations (as well as constraints of planning and initiating eye movements), but also that these conceptual operations may be distributed over later fixations and even postponed until a syntactic boundary is reached.
\end{abstract}

For most of us, rapid reading involves some form of skimming in which we try to focus on information relevant to our goal and skip over irrelevant information. In fact, studies of eye movements of readers who are given the task of rapidly processing text have shown that readers will fail to view parts of a text while reading others in a fashion that resembles normal reading (Taylor, 1962; Just, Carpenter, \& Masson, Note 1). It is also true, however, that according to comprehension tests readers are not very accurate at visually selecting goal-relevant information for processing (Masson, 1982; Masson, Carpenter, \& Just, Note 2). Important information is often missed while a good deal of irrelevant information is sampled.

Given the apparent inefficiency of this method of rapid reading, it is reasonable to ask why other methods that guarantee exposure to all information in a text are not commonly used. For example, when readers wish to increase their processing rate, why do they not simply reduce the duration of each fixation (the average fixation during normal reading is $200-250 \mathrm{msec}$; see Rayner,

The research reported here was supported by Grant A7910 from the Natural Sciences and Engineering Research Council of Canada and by grants from the University of Victoria. I am grateful to Chris Carlson, Geoff Espin, Nancy Letkeman, Ann Lloyd, Linda Low, Barbara Oman, and Brad Piekkola for their assistance in collecting and scoring data. A preliminary report of Experiments 1-3 was presented at the annual meeting of the Psychonomic Society, Philadelphia, Pennsylvania, November 1981. A preliminary report of Experiments 4 and 5 was presented at the meeting of the Canadian Psychological Association, Montreal, Canada, June 1982. Requests for reprints should be sent to Michael Masson, Department of Psychology, University of Victoria, Victoria, British Columbia V8W 2Y2, Canada.
1978; Rayner \& McConkie, 1976) and ensure that every word is seen? Total time spent on each word, cumulated over successive fixations, actually is reduced during skimming, but not by an amount large enough to permit doubling or tripling of reading rate without skipping material (Just et al., Note 1).

One reason that readers do not drastically reduce fixation durations to achieve high reading speeds may be that the oculomotor system responsible for controlling eye movements is not sufficiently adroit to make the required series of very brief, accurately placed fixations. Studies mimicking eye movement patterns employed during reading have shown that an average of about $175-200 \mathrm{msec}$ is required for each of a series of fixations made at targeted locations, even though no conceptual processing of targets was required (Arnold \& Tinker, 1939; Salthouse \& Ellis, 1980). Moreover, evidence from eye movement and perceptual span studies indicates that conceptual processing of a word requires foveal fixation and only about one word at a time can be so fixated (Rayner, 1975; Rayner \& McConkie, 1977). The time demands of the oculomotor system and the size of the perceptual span would place severe upper bounds on the rate at which a text can be processed if every word is to be fixated.

A second reason that increased reading speed might not be obtainable by reducing fixation durations is that conceptual processing of fixated information may require a minimum amount of time approximating the average fixation duration. Salthouse, Ellis, Diener, and Somberg (1981) have shown that conceptual processing of a simple visual stimulus (e.g., identity of the stimulus determines the direction of the next saccade) often 
extends at least as far as the first $200 \mathrm{msec}$ of a fixation. In the context of a reading task, Just and Carpenter (1980) found that time spent fixating individual words is related to conceptual characteristics of the words, such as frequency and grammatical case, indicating that additional viewing time is necessary for the completion of more complex conceptual processes. The reluctance of readers to abbreviate fixations during reading might, therefore, be partly due to the potentially disastrous consequences for conceptual processing of text. That is, comprehension processes such as lexical access, retrieval of information from long-term memory, inference making, and creating new text-based representations in memory may require more time than would be available if reading speed were increased by a drastic reduction of eye fixation durations (cf. Gibson \& Levin, 1975; Poulton, 1958, 1963; Sticht, 1977).

Average fixation durations would be much longer than they actually are if eye movement control and conceptual processing time combined additively to determine fixation duration. Consequently, it has been suggested (e.g., Rayner \& Pollatsek, 1981; Salthouse et al., 1981) that unconscious "planning" of the next eye movement and conceptual processing may overlap in time during a fixation. To help assess the relative contribution of these two constraints on fixation duration, both Rayner and Pollatsek and Salthouse et al. have pointed to results obtained using rapid sequential visual presentation (RSVP) of words.

The traditional rapid sequential reading procedure involves presentation of text one word at a time, for a brief duration at a single visual location. This procedure eliminates the need to make eye movements and should, therefore, allow a rather pure assessment of the time course of conceptual processing of words during reading. If the duration of exposure to each word is reduced too much, comprehension should suffer markedly. An argument against the use of this technique that immediately crops up is the complaint that if presentation duration is too short, masking effects, rather than conceptual processing time constraints, might reduce reading performance. This argument loses much of its force in light of Rayner, Inhoff, Morrison, Slowiaczek, and Bertera's (1981) finding that readers are capable of extracting the visual information necessary for reading within the first $50 \mathrm{msec}$ of a fixation. They presented a mask to readers at varying delays after the onset of a fixation during reading. When the mask was delayed by $50 \mathrm{msec}$ or more, characteristics of reading performance such as duration and number of fixations were similar to those obtained when no mask was presented. Rayner et al. concluded that the extra time used in each fixation is required for conceptual processing of what was seen and for programming the next eye movement. There is, however, a tradeoff in this counterargument involving the weak masking effects of a blurred pattern seen during a saccade in normal reading compared to the masking effects of the clear pattern of the succeeding word in RSVP.

The importance of this tradeoff is reduced by recent results involving very brief RSVP durations. Juola, Ward, and McNamara (1982) presented subjects single RSVP sentences with the goal of determining whether a sentence contained a word that belonged to a predesignated semantic category (e.g., food). Even when the duration of each word in a target sentence was as short as $50 \mathrm{msec}$, subjects were correct on about $90 \%$ of the trials. In addition, Fischler and Bloom (1980) have shown that with RSVP durations as low as $36 \mathrm{msec} /$ word, sentence contexts can influence lexical decisions about target items that represent semantically anomalous completions of the contexts. This result has been interpreted as evidence that long-term memory information concerning the sentences' propositions had been activated.

More relevant to the task of text processing, Potter, Kroll, and Harris (1980) have reported impressive evidence concerning comprehension of paragraphs presented under rapid sequential reading conditions. They used paragraphs of the same genre as those used by Bransford and Johnson (1972) and Dooling and Lachman (1971). The passages were grammatically correct but semantically ambiguous and poorly integrated unless a special topic word was included in the passage. For instance, one passage included the statement, "I pulled at one section, but it was difficult to remove, so I tried another," without providing any clue to the identity of "it." The passage was made quite comprehensible, however, by including the topic word "pizza." Potter et al. manipulated whether and where the topic word appeared in the passages and presented them in RSVP and whole-passage formats. In the RSVP condition, they used presentation durations ranging from 83 to $250 \mathrm{msec} /$ word, with a pause between each sentence that was equivalent to the duration of two words. Recall performance in this condition was compared with that of a skimming condition in which passages were presented in their entirety for a limited time equivalent to the time required to present the passages in the RSVP condition.

Mention of the topic word was taken as a measure of comprehension, and superior performance was found in the RSVP compared to the skimming condition, especially at rapid reading rates and when the topic word appeared late in the passage. In fact, mentioning the topic was affected very little by increased reading rate in the RSVP condition, although recall suffered markedly. It was concluded that comprehension processes (as assessed by topic mention) were capable of keeping pace with the rapid rate of text presentation, although subjects were not able to incorporate all the information into long-term memory. This conclusion is similar to that reached in work with rapid sequential presentation of pictures, for which it has been shown that subjects 
can accurately detect the presence of a verbally predesignated picture but do poorly on a recognition test given after presentation of a series of pictures (Intraub, 1981; Potter, 1976).

The results of these experiments imply that readers may indeed be capable of comprehending text when it is presented in a series of short glimpses that last for much less time than the average eye fixation. Results of this sort have also been taken as evidence that comprehension processes under RSVP are very similar to those that operate when reading conventionally presented text (e.g., Juola et al., 1982). There are, however, several qualifications that should be applied to these conclusions. First, the quality of comprehension associated with accurate performance in a detection task or with priming of lexical decisions is rather different from the level of comprehension involved in the integration of a sentence's words and propositions into meaningful ideas.

Potter et al.'s (1980) work with RSVP and skimming provided a much closer approximation to sentence and text comprehension. But performance of subjects in their RSVP condition might have been exaggerated, relative to performance in the skimming condition, through the use of unusually constructed passages. Recall and topic report depended critically on processing the topic word, and only subjects in the RSVP condition were guaranteed exposure to the word. In the skimming condition, subjects failed to see a significant portion of the words (from the second half of the passage because of slow reading rate, or from arbitrarily selected sections due to skipping) and so easily could have completely missed the topic word.

Therefore, potential text comprehension and memory problems associated with abbreviated glimpses of text might have been obscured. Normally constructed passages might be well understood and remembered by skimmers, but special difficulties might arise when passages are presented using RSVP, even though readers are exposed to all of the text information in this case. The first three experiments reported here were designed to test this possibility using a variety of comprehension and memory tests.

\section{EXPERIMENT 1}

The first experiment consisted of a replication of the comparison of skimming and rapid sequential reading previously made by Potter et al. (1980). Unlike Potter et al.'s study, the passages were selected from a conventional source (Reader's Digest) and no pauses were inserted between sentences. Potter et al. included intersentence pauses to help subjects distinguish sentence boundaries, but the use of such pauses takes on added significance, as will be seen when Experiments 4 and 5 are described. For the purposes of the first three experiments, it was decided to allow capitalization and periods to signal sentence boundaries.
Text memory and comprehension were evaluated through question answering. The use of this technique leaves open the possibility that questions may successfully be answered either as a result of correctly comprehending and encoding into long-term memory the relevant information during reading or as a result of identifying only a few critical words during reading and basing an answer on a partially correct reconstruction of the relevant information. I would argue that while it is difficult to determine which of these two processes are responsible for answering a question (just as it is difficult to determine which is responsible for allowing recall or recognition of text content), logic dictates that there should be a strong relationship between question answering accuracy and type of process involved. Specifically, identifying only a few critical words and relying on postreading reconstruction is bound to produce less accurate answers and also reflects poorer quality comprehension (e.g., Bartlett, 1932). Therefore, question answering performance should be a valid method of assessing the relative accuracy and completeness of text comprehension and memory.

Two questions were developed for each passage, one relevant to the general topic of the passage and the other relevant to a specific detail. By using these two question types, it was expected that subjects' understanding and memory of different levels of passage content could be assessed. For example, if conceptual processing of individual words consistently requires more time than is available during a word's presentation, subjects should have difficulty integrating the words into coherent idea units or propositions. Under these circumstances, answering either type of question should be difficult. Alternatively, if subjects are able to extract enough conceptual information from words, they may be able to understand individual sentences, but they might have trouble integrating information across sentences. This would result in relatively poor performance on general questions.

Finally, if comprehension at all levels can keep pace with the rapid visual input involved in RSVP and if information is adequately encoded into memory, rapid sequential reading should lead to a more complete memory representation and more accurate question answering than should skimming. This is because skimmers characteristically fail to visually sample significant amounts of the text (Just et al., Note 1). This fact, however, suggests an important limitation of the use of skimming as a baseline for comparison against RSVP performance. Comparing these two rapid reading tasks involves a tradeoff between fixation duration and amount of text sampled, which complicates the assessment of the effects of abbreviated fixation durations. In addition, it is difficult to know exactly how skimmers distribute their reading time across the different parts of a passage. Skimming is used as a baseline in these experiments primarily to make contact with previous literature.

In order to increase the likelihood of subjects' being 
able to encode information into memory during RSVP only moderately fast reading rates of 375 and 500 words/ min (wpm) were used, the latter being very similar to Potter et al.'s (1980) second fastest rate.

\section{Method}

Subjects. Forty-eight undergraduate students at the University of Victoria served in the experiment on a voluntary basis. Half of the subjects read the passages at $375 \mathrm{wpm}$, and half read at $500 \mathrm{wpm}$. An additional 24 students who did not read any of the passages attempted to answer the comprehension questions on the basis of their general knowledge.

Materials. Forty short passages (mean length $=133$ words) were selected from unrelated Reader's Digest stories. Two questions, which could be answered with a short phrase or a single word, were prepared for each story. One queried information directly relevant to the main theme of the passage, and the other asked about a less important detail. For example, a passage about Montgomery Ward inventing the mail-order catalog business was followed by the general question, "What new business did Ward establish?", but the specific question was "Prior to establishing his business what was Ward's occupation?" (he was a traveling salesman). The questions were designed so that the wording of one question would not reveal the answer to the other question.

Procedure. Presentation of the passages was accomplished using a 23-cm (diagonal) black-and-white Electrohome video monitor and an Apple II microcomputer equipped to display 80 upper- and lowercase characters on each of 24 lines. For the skimming condition, each passage appeared in its entirety in the center part of the monitor's screen. In the rapid sequential reading condition, words appeared individually, in serial order, at the center of the screen. The first letter of each word appeared in the same location. When a word's presentation duration elapsed, the word disappeared and was immediately replaced by the next word. Words that began a sentence were capitalized, and a period appeared beside the last character of each word that ended a sentence.

Subjects read 20 consecutive passages in the skimming condition and 20 in RSVP. Order of display format and assignment of passages to display format were counterbalanced across subjects. Instructions introducing the display format preceded each set of 20 passages, and the first 4 in each format were treated as practice items. For the skimming condition, subjects were told to skim the whole passage in the limited time that it would be on the screen. Passages stayed on the screen for a fixed time period determined by the subject's reading rate condition and the mean length of the passages (i.e., $21.28 \mathrm{sec}$ in the 375-wpm condition and $15.96 \mathrm{sec}$ in the $500-\mathrm{wpm}$ condition). Although the critical passages ranged in length from 101 to 151 words, the discrepancy between the standard presentation duration in the whole-passage condition and the duration that would be dictated by individual passage length was greater than 2 or $3 \mathrm{sec}$ (in the $500-$ and $375-\mathrm{wpm}$ conditions, respectively) for only eight passages.

In the rapid sequential reading condition, subjects were told about the nature of the presentation format and to try to understand the whole passage. Each word of a passage was presented for a fixed duration, depending on the subject's reading rate condition (i.e., $160 \mathrm{msec}$ in the 375 -wpm condition and $120 \mathrm{msec}$ in the 500 -wpm condition), with no pauses between words. Properties of the raster scan monitor introduced some variation in the actual duration of presentation of each word (Reed, 1979), but the total time required to present a passage in RSVP was highly consistent and accurately controlled. After reading each passage, subjects were presented first the general and then the specific question for that passage, and they recorded their answers on paper. Subjects worked at their own pace, initiating presentation of each passage and question through buttonpresses. The 24 control subjects were given printed copies of the 80 questions and attempted to answer them without having read the passages.

\section{Results and Discussion}

Answers to questions were scored by assigning 1 point to fully correct answers, .5 point to partially correct responses, and 0 points to incorrect answers and omissions. Accurate paraphrases were accepted as fully correct responses. Credit was given for partially correct answers when incomplete information was supplied. For example, in answering the question about Montgomery Ward's early career as a traveling salesman, claiming only that he was a salesman was considered partially correct. The questions associated with the eight practice passages were not included in the scoring. Using this method, each subject obtained a proportion correct score on general and specific questions, in each of the two reading format conditions. Mean question answering scores for the three groups of subjects are shown in Figure 1.

When data from subjects who read the passages are considered, a general advantage for the slower reading rate and for general questions is apparent. In addition, there is a clear advantage for the whole passage format over RSVP, at least for answering general questions. These impressions were confirmed by an analysis of variance that included reading rate, format, and question type as factors (alpha error in this and other analyses was set at .05). All three main effects were significant,

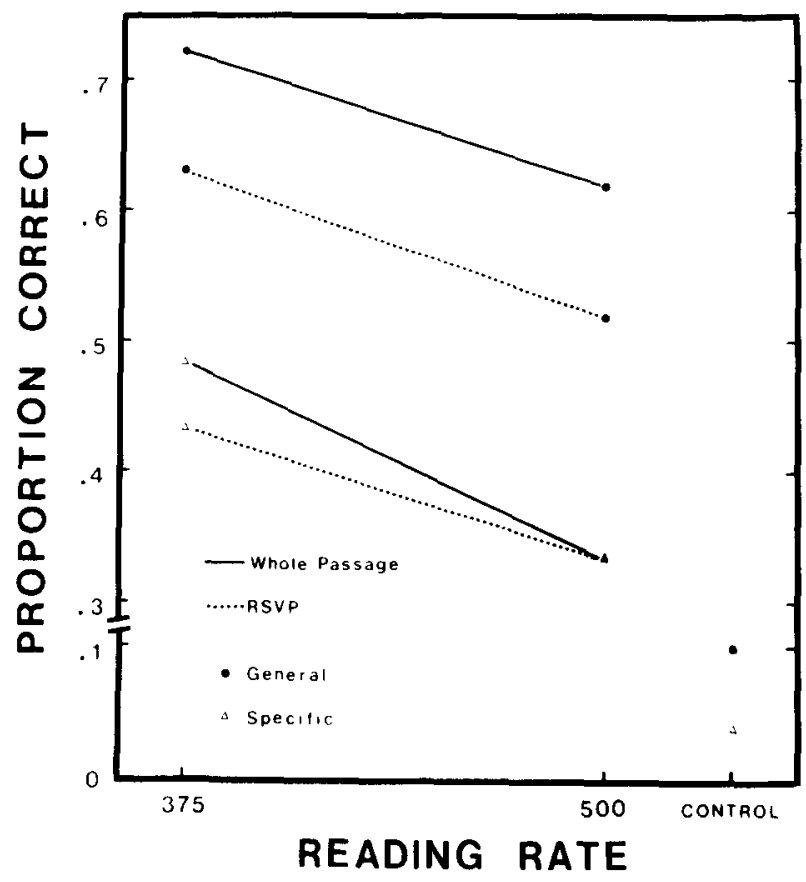

Figure 1. Mean proportion of correctly answered questions in Experiment 1. 
indicating that questions were more accurately answered when the slower reading rate was in effect $[F(1,46)=$ $7.37, \mathrm{MSe}=.085]$ and when passages were presented in conventional as opposed to RSVP format $[F(1,46)=$ $8.10, \mathrm{MSe}=.020]$ and that general questions were more accurately answered than specific ones $[F(1,46)=$ 130.31, $\mathrm{MSe}=.019$ ]. In addition, there was a reliable interaction involving reading format and question type $[F(1,46)=4.95$, MSe $=.013]$, suggesting that most of the reading format effect was due to performance on general questions.

Separate analyses of variance comparing subjects' performance under each reading format to the performance of subjects who never read the stories were conducted to determine whether the question type effect was produced by selective processing of general information in the passages or was simply due to preexperimental knowledge about the passage topics. If the effect was due to selective processing of passage gist, these analyses should produce an interaction between reading group ( $375 \mathrm{wpm}, 500 \mathrm{wpm}$, and control) and question type. As can be seen from Figure 1, the question type effect is rather small among control subjects and the two analyses of variance verified this observation. Both analyses (a counterbalanced selection of items was used for each control subject to make the arbitrary reading format distinction) revealed a reliable interaction between group and question type $[F(2,69)=$ $12.03, \mathrm{MSe}=.014$, for the whole-passage analysis, and $\mathrm{F}(2,69)=7.21, \mathrm{MSe}=.010$, for the RSVP analysis $]$.

It might be argued that the advantage observed in the skimming condition was due to a reading strategy similar to that adopted by many of Potter et al.'s (1980) subjects in which reading time was concentrated on the first part of each passage. To test this possibility, the following analysis was done. The distance into the passage that one would have to read in order to correctly answer a question was determined for each test item. The correlation between this distance measure and the proportion of subjects correctly answering a question was calculated for eight different cases defined by the possible combinations of reading rate, format, and question type. A significant negative correlation would indicate that subjects were most often able to answer items appearing early in the passages, indicating that they were reading only the early portions of the passages rather than covering the entire length. The only coefficient that reached significance involved specific questions in the skimming condition at $500 \mathrm{wpm}[\mathrm{r}(30)=$ $-.61]$, and the remaining coefficients were less extreme than -.26 .

An analysis of performance on questions whose answers appeared in the first vs. the second half of a passage corroborated these results. As can be seen in Table 1, the greatest drop in performance across a passage occurred for specific questions in the $500-\mathrm{wpm}$ group when skimming. In all other cases, performance
Table 1

Mean Proportion of Questions Answered in Experiment 1 as a Function of Location of the Answer in the Passage

\begin{tabular}{clcccc} 
& & \multicolumn{4}{c}{ Reading Rate } \\
\cline { 3 - 6 } Question & Reading & \multicolumn{3}{c}{$375 \mathrm{wpm}$} & \multicolumn{2}{c}{$500 \mathrm{wpm}$} \\
\cline { 3 - 6 } Level & Format & 1 & 2 & 1 & 2 \\
\hline General & Skimming & .77 & .68 & .67 & .58 \\
General & RSVP & .69 & .56 & .57 & .52 \\
Specific & Skimming & .50 & .45 & .44 & .24 \\
Specific & RSVP & .44 & .42 & .33 & .34 \\
\hline
\end{tabular}

Note-1 = answer was located in the 1st half; 2 =answer was located in 2nd half.

decrements across a passage were similar for skimming and rapid sequential reading conditions. These results strongly argue against the idea that the advantage observed in the skimming condition was due to concentration of reading time on only the first half of a passage, and they also suggest that in the case of skimming at $500 \mathrm{wpm}$, subjects processed gist-relevant information throughout the passage while attending to detailed information primarily during the first part of the passage.

The finding that skimming led to better question answering performance (at least on gist-relevant items) than rapid sequential reading is consistent with the hypothesis that abbreviated glimpses of every word in a text alters comprehension processes to a significant degree, relative to the case in which readers use natural eye movements to skim a passage. Potter et al. (1980) probably failed to find evidence of this sort because of their use of specially constructed passages that depended on readers' making use of a critical topic word embedded in each passage. When more conventional materials are used, skimmers appear to have an advantage, at least with respect to comprehension and memory of the gist of a passage. Contrasting with this conclusion, however, is Juola's (Juola et al., 1982; Juola, Cocklin, Chen, \& Granaas, Note 3) finding that answering multiple-choice questions was equally accurate after skimming and RSVP reading. But his failure to find any differences likely was due in large part to the fact that his RSVP conditions typically involved 200 - or $300-\mathrm{msec}$ presentations of small groups of words at a fixed location on a screen. This presentation method is virtually a simulation of skimming, in that fixation durations are not drastically reduced and a subject is able to foveally process only a portion of each displayed text segment.

\section{EXPERIMENT 2}

The disadvantage of rapid sequential reading, relative to skimming, found in Experiment 1 strongly implies that readers are not able to fully comprehend and encode into memory texts that are presented through a series of abbreviated fixation durations. Since deficits 
were apparent only when general questions were considered, Experiment 2 was designed to further explore the issue of potential macrostructure processing difficulties associated with RSVP. Rather than answering questions, subjects were asked to provide a single topic sentence that described the main idea of a passage. Kieras $(1980,1981)$ has shown that this task is a sensitive measure of a reader's success in constructing an accurate macrostructure representation of a passage. The objective was to encourage subjects to focus their comprehension processes on gist-relevant information. It was expected that if the performance deficit observed in Experiment 1 was at least partly due to poor integrative processing of ideas across sentences during rapid sequential presentation of text, subjects probably would not be able to make the best possible use of the visual information available to them to generate a representative text macrostructure. When skimming, readers do not seem to spend very different amounts of time viewing gist-relevant as opposed to irrelevant information (Masson, 1982; Just et al., Note 1), but their conceptual processing of sampled information is sufficiently selective to produce acceptable macrostructure representations (Masson, 1982; Masson et al., Note 2).

\section{Method}

Subjects. Forty-eight subjects were recruited from the source used in Experiment 1, and 24 were assigned to each of two reading rate conditions.

Materials. The 40 passages from Experiment 1 were used. The main idea contained in each passage was empirically determined by having a group of 20 subjects read each passage for unlimited time and provide one-sentence summaries of each. The summaries were divided into idea units that roughly corresponded to clauses. Each idea unit mentioned in at least 1 of the 20 summaries of a passage was assigned a value that represented the proportion of summaries in which the idea unit was mentioned. These values were used to assess the accuracy or representativeness of the summaries produced by subjects who read the passages under skimming and rapid sequential reading conditions.

Procedure. The procedure was identical to that used in Experiment 1, except that no questions were asked after the passages were read. Subjects were instead required to write one sentence that summarized the main idea of a passage immediately after reading it. They were instructed that this was their only task and that they should read the passages with the task in mind. As in Experiment 1, the first four passages read under each presentation format were treated as practice passages and were not included in the scoring.

\section{Results and Discussion}

The degree to which the summaries produced by the rapid readers accurately reflected the main ideas of the passages was determined by segmenting the summaries into idea units, as was done for the 20 control subjects who were given unlimited reading time. Each idea unit in a subject's summary was assigned a score that was equal to the proportion of control subjects who included that idea unit in their corresponding summaries. The overall score for a subject's summary was simply the mean of the scores of the idea units contained in the summary. Consequently, a high score could be obtained only when all idea units in a summary were consistent with idea units most frequently mentioned by the control subjects and, therefore, reflected strong agreement with the control subjects' interpretation of the main idea of a passage. Including unimportant details or omitting important information reduced the value of a summary.

This system of evaluating summaries places a ceiling on scores that is dependent on the level of agreement among control subject's summaries. Specifically, when the idea unit most frequently mentioned by control subjects was selected from each of the 32 critical passages, the mean proportion of agreement score for these idea units was .57 . So if a subject was to include only the most important idea unit in each of his or her summaries, the maximum score that subject could receive would be .57 . The proportion agreement scores reported below should be interpreted with this ceiling in mind.

The mean proportion agreement scores are shown in Figure 2. An analysis of variance of these data indicated that summaries were more accurate in the skimming condition than in the RSVP condition $[F(1,46)=9.80$, $\mathrm{MSe}=.002]$ and that more accurate summaries were produced when reading more slowly $[F(1,46)=14.15$, $\mathrm{MSe}=.006]$. The interaction between these variables approached significance $[F(1,46)=3.49, p<.07]$, suggesting that the effect of reading format was stronger when subjects read faster. The advantage observed in the skimming condition was not due to subjects' including different amounts of information in their summaries relative to the RSVP condition. Total numbers of idea units included in summaries were almost identical in the two conditions (1.75 in the skimming condition and

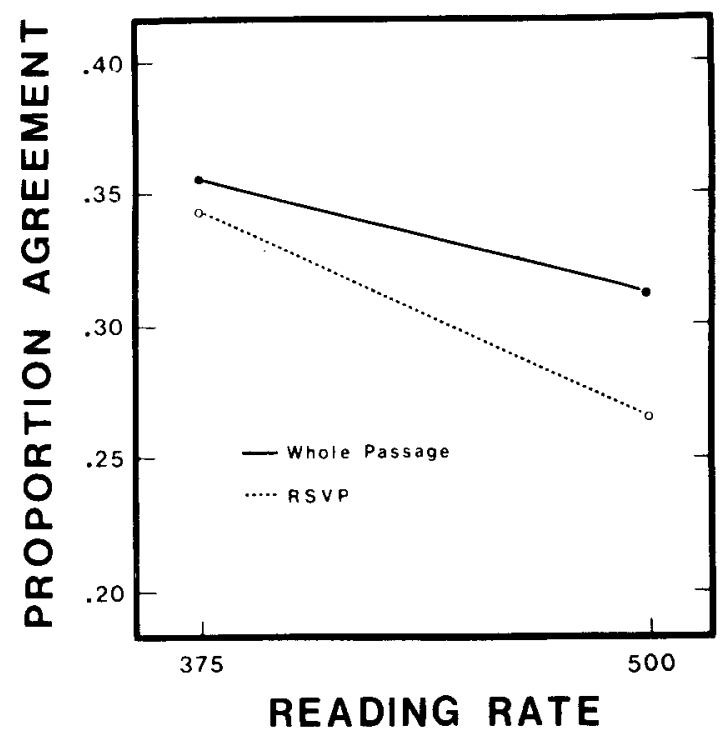

Figure 2. Mean proportion of agreement between rapid readers and careful readers on idea units included in passage summaries in Experiment 2. 
1.73 in the RSVP condition). Moreover, these results need not be strongly qualified by the argument that subjects had a special advantage when skimming because topic-relevant information occurs early in a passage. In fact, when the two most important idea units for each passage were considered, the mean number of lines into a passage one would have to read in order to obtain the relevant information was 4.8 . Since the passages were 10-12 lines long, it is clear that a good deal of topic-relevant information appeared in the second half of the passages.

These results support the claim that identifiable conceptual processing difficulties arise when subjects are provided with brief glimpses of text rather than being allowed to spend relatively long periods of time with each segment. Readers at least have difficulty developing an accurate representation of the gist of passages read under RSVP. It is also possible that RSVP makes comprehension of detailed information more difficult as well, although no direct evidence for this has yet been offered. But if subjects in Experiment 1 had traded off processing of gist information in favor of obtaining more detailed information, we would have obtained evidence for problems at both levels of comprehension during RSVP. Therefore, it would be unwise at this stage to conclude that abbreviating fixation durations produces deficits only at the level of gist comprehension.

It is unclear, however, whether such difficulties arise as a result of comprehension inefficiency or of longterm memory encoding failure (cf. Ortony, 1978). Potter et al. (1980) have suggested that subjects are able to at least momentarily understand text as it is presented during rapid sequential reading, although they are not able to accurately encode the information into long-term memory. To test this possibility, a variant of the question answering task was used in Experiment 3. Rather than probing subjects' memory for the passages, a single question was presented before each passage was read. The subject's only task was to comprehend the passage so that the best possible answer to the question could be obtained. This task is very much like a target detection task (cf. Intraub, 1981; Juola et al., 1982, Experiment 3), except that the target is conceptually rather complex. Subjects should be able to accurately answer predesignated questions if comprehension during RSVP reaches a sufficiently high level (e.g., goes beyond the activation of particular regions of long-term memory demonstrated by Fischler \& Bloom, 1980).

When skimming, however, subjects are not guaranteed exposure to the relevant parts of a passage and must therefore scan for the appropriate information. The tradeoff in this case is the possibility of failing to find the target information vs. being able to spend a relatively long time reading that information when it is found. The task of skimming to find the answer to a predesignated question may, however, be less than perfect as a baseline for comparison with RSVP, particularly when detailed questions are considered. This is because skimmers may be able to scan a text for key words that were included in the predesignated question and then carefully read the surrounding information to obtain the answer. This strategy would be less useful in the case of gist questions, since the answer to these items requries consideration of information from a number of different locations in a passage.

Faster reading rates were used in Experiment 3 than in the earlier experiments, since it was expected that answering predesignated questions would be much easier than answering questions from memory.

\section{EXPERIMENT 3}

\section{Method}

Subjects. Forty-four students drawn from the pool used in the first two experiments particpated in Experiment 3. Half were assigned to each of two reading rate conditions.

Materials and Procedure. The passages and questions from Experiment 1 were used in Experiment 3 . The procedure was identical to that used in Experiment 1, except that only one question was asked about each passage and the question was shown to the subject before and after the passage was read. Subjects were told that their only task was to find the answer to the given question by reading the passage. Upon seeing the question on the second occasion. the subject recorded his or her answer on a piece of paper. Half of the passages read in each format were queried by a general question, and half were queried by a specific question. Type of question asked about a passage and assignment of passages to reading format condition were counterbalanced across subjects. Half of the subjects read the passages at the equivalent of $500 \mathrm{wpm}$, and half read at $700 \mathrm{wpm}$.

\section{Results and Discussion}

Question answering was scored as in Experiment 1, and mean scores are shown in Figure 3. Also presented is the mean performance of 22 of the 24 control subjects who attempted to answer the questions without having read the passages. The control subjects' scores were based on a counterbalanced selection of questions that corresponded to questions answered by the subjects who read the passages in Experiment 3. An analysis of variance of the scores of subjects who read the passages indicated that performance was reliably better when subjects skimmed whole passages than when they read under RSVP $[F(1,42)=19.61, \mathrm{MSe}=.056]$ and that answers were more accurate for subjects in the slower reading rate condition $[\mathrm{F}(1,42)=18.46, \mathrm{MSe}=.051]$. There was a marginally significant interaction between reading rate and format $[F(1,42)=3.99, p<.06]$, suggesting that the skimming condition advantage increased with reading rate. There was also a marginally reliable interaction between reading format and question level $[\mathrm{F}(1,42)=3.13, \mathrm{MSe}=.017, \mathrm{p}<.09]$.

In order to determine whether performance differences on general vs. specific questions in either of the reading formats reliably exceeded differences among 


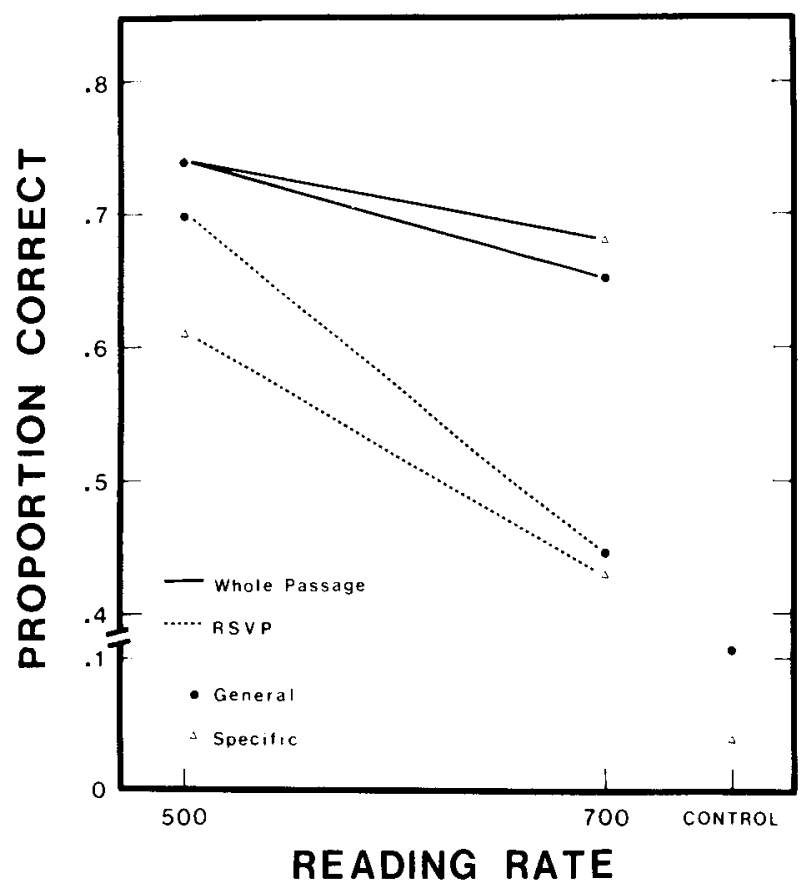

Figure 3. Mean proportion of correctly answered questions in Experiment 3.

control subjects, two separate analyses of variance were carried out involving the 22 control subjects. In the analysis involving control subjects and performance in the whole-passage condition, there was no interaction between question type and reading group $(500 \mathrm{wpm}$, $700 \mathrm{wpm}$, and control), indicating that readers were able to comprehend answers to general and specific questions about equally well. The same result was found when the analysis involving control subjects and performance in the RSVP condition was carried out.

There was a tendency for subjects to answer questions occurring earlier in the passages more accurately, but this was true only in the 700-wpm condition. Significant correlations between location of the answer to a question and probability of correctly answering it were found when general and specific questions in this condition were considered ( $\mathrm{rs}=-.40$ and -.50 , respectively).

Comprehension, as assessed by the ability to answer predesignated questions, was markedly superior when subjects skimmed passages compared to when they read under RSVP. It appears that the level of comprehension attained during rapid sequential reading may often not involve conscious integration of elements of text information with themselves or with relevant general knowledge. Although subjects risked skipping relevant information when skimming, they were able to locate enough information to consistently answer questions even when passages were available for the short time corresponding to the $700-\mathrm{wpm}$ condition. In addition, when relevant information was located, subjects would be able to read it in a manner more consistent with normal reading than would be the case for RSVP, thereby ensuring more complete comprehension. The comprehension difficulties encountered during rapid sequential reading were not restricted to gist-relevant information, but they were at least as great for detailed information. This outcome might have been anticipated, since skimmers have a particular advantage when searching for the answer to a specific question (visual search often can be restricted to a small set of key words and to a single location in the passage).

The comparison between skimming and RSVP performance provided some evidence that abbreviating fixation durations through rapid sequential reading reduces the ability to comprehend text as it is being presented. By combining results from Experiments 1 and 3 , however, it is possible to assess long-term memory encoding deficits associated with RSVP, without resorting to comparisons between skimming and RSVP performance. The rapid sequential reading performance of subjects in Experiments 1 and 3 who read passages at $500 \mathrm{wpm}$ was submitted to an analysis of variance (two subjects from Experiment 1 were randomly chosen for exclusion to equalize sample sizes). Subjects in Experi- ment 1 answered questions that were seen for the first time only after a passage had been read and, therefore, relied heavily on long-term memory encoding of information from the passages. By comparison, searching for answers to predesignated questions in Experiment 3 (in which immediate comprehension would be sufficient to accurately answer a question) led to reliably better performance $(.655$ vs. .421$)[\mathrm{F}(1,42)=25.12$, MSe $=$ $.048]$. General questions were more accurately answered than specific ones $(.607$ vs. .469) $[F(1,42)=17.07$, MSe $=.025]$. The interaction was not reliable $(p>.14)$. This pattern of results indicates that during rapid sequential reading, subjects had difficulty encoding into long-term memory sufficient text information to answer either gist or detailed questions.

\section{EXPERIMENT 4}

The results of the first three experiments strongly imply that serious conceptual processing problems may arise when readers attempt to comprehend text presented under RSVP. The possible role of perceptual difficulties associated with RSVP in producing the deficits observed here has been set aside primarily on the grounds of previous research. Therefore, in order to reinforce the claim that the comprehension and memory problems found with RSVP derive from conceptual processing limitations, Experiments 4 and 5 were designed to test the idea that it should be possible to alter the presentation of RSVP passages in a manner that would improve conceptual processing while changing the perceptual characteristics of the presentation mode only slightly. 
Two classes of reading models provide a suggestion concerning how RSVP might be altered to promote comprehension and memory encoding. Cyclic processing models such as those proposed by Kintsch (Kintsch \& van Dijk, 1978; Miller \& Kintsch, 1980) and Perfetti and Lesgold (1977) are based on the assumption that consecutive segments of a text are processed in working memory in successive cycles. Similarly, buffer models (e.g., Green, Mitchell, \& Hammond, 1981; Mitchell \& Green, 1978) work on the assumption that information from a sentence or a clause is stored in a limited-capacity buffer until, for example, a relevant syntactic boundary is reached; then processing is concentrated on the contents of the buffer. When information is in the processing cycle or buffer it is intergrated with earlier information and is used to generate inferences that contribute to the coherence of the text. If a new text segment is forced into the system before completion of a processing cycle or before the information already in the buffer has been processed, the reader may fail to integrate the pending information with earlier material and may also fail to accurately encode the intruding information.

The need for buffering information might be greater during rapid sequential than during normal reading because the brief glimpses of words in the former case may abbreviate fundamentally important comprehension processes, including lexical access (cf. Just, Carpenter, \& Woolley, 1982; Thibadeau, Just, \& Carpenter, in press). Therefore, altering RSVP to promote the processing of buffered information should lead to improved rapid text processing. This claim was tested by inserting pauses between sentences during rapid sequential reading. Besides merely signaling sentence boundaries, these pauses were expected to cue and provide an opportunity for subjects to focus conceptual processing on information that may have been buffered during a sentence's presentation.

This change, however, involves only a minor deviation from the perceptual characteristics of the RSVP procedure used in Experiments 1-3, as the last word of each sentence has a longer delay before the onset of its mask (i.e., the next word) and there is limited forward masking of the first word of each sentence. The associated changes in perceptual processing should not be responsible for bringing about noticeable improvement in the comprehension of a whole passage.

Experiment 4, then, was a replication of Experiment 1 including a condition in which a pause of $500 \mathrm{msec}$ was inserted between each sentence during RSVP. It was expected that if buffering segments of a passage was particularly important during RSVP and that if intersentence pauses cued and provided sufficient opportunity for such operations, the presence of the pauses should reduce or eliminate the difference between skimming and RSVP conditions on the question answering task. This outcome would be consistent with results obtained by Juola et al. (1982) and Potter et al. (1980) involving recall and question answering after skimming and rapid sequential reading. In both studies, pauses were inserted between sentences, and when conventional passages were used, no differences between skimming and RSVP performance were observed.

\section{Method}

Subjects. A sample of 48 subjects was drawn from the pool used in earlier experiments. Half were assigned to a condition in which pauses were inserted between sentences during RSVP and half to a condition that did not include pauses.

Materials and Procedure. The materials and procedure were identical to those of Experiment 1, with the following exceptions. The materials were presented on a $30-\mathrm{cm}$ (diagonal) green Zenith video monitor as opposed to the smaller black-and-white monitor, and the middle letter of each word in the RSVP condition appeared in a fixed location. As in the earlier experiments, subjects read half the passages in conventional format and half in RSVP. For half of the subjects, sentences presented in RSVP followed immediately after each other, with each word presented for $100 \mathrm{msec}$, making an overall rate of $600 \mathrm{wpm}$. These subjects were allowed viewing time in the whole-format condition that corresponded to a reading rate of $600 \mathrm{wpm}$. For the remaining subjects, words were presented for a duration of $100 \mathrm{msec}$ each, but a pause of $500 \mathrm{msec}$ was inserted between sentences, during which the screen was blank. Given the average number of sentences in the passages, the pauses produced an overall reading rate of $500 \mathrm{wpm}$. Therefore, these subjects were allowed viewing time in the whole-passage format condition that corresponded to that rate. The average viewing times in the whole-passage condition were 13.30 and $15.96 \mathrm{sec}$. Unlike earlier experiments, however, viewing time in the whole-passage condition depended on the length of the specific passage rather than on the mean length of 133 words.

\section{Results and Discussion}

Question answering was scored as in the earlier experiments, and mean performance of the two groups of subjects is shown in Table 2. An analysis of variance of these data confirmed the prediction that intersentence pauses would improve RSVP performance relative to skimming, at least if a one-tailed test of significance is applied. Given the specificity of the prediction, the use of a more lenient criterion for significance seems justified. There was a main effect of reading format in which skimming led to more accurate question answering than did rapid sequential reading $[\mathrm{F}(1,46)=14.08$, $\mathrm{MSe}=.012]$, but there was also a marginally significant interaction between reading format and group $[F(1,46)$ $=2.83, p<.10(p<.05$ by a one-tailed test $)]$, indicating that the reading format effect was reduced when a

Table 2

Mean Proportion of Questions Answered in Experiment 4

\begin{tabular}{clccc}
\hline & \multicolumn{4}{c}{ Reading Rate and Format } \\
\cline { 2 - 5 } $\begin{array}{c}\text { Question } \\
\text { Level }\end{array}$ & \multicolumn{2}{c}{$600 \mathrm{wpm}$} & \multicolumn{2}{c}{$500 \mathrm{wpm}$} \\
\cline { 2 - 5 } & WP & RSVP & WP & RSVP+P \\
\hline General & .395 & .311 & .394 & .355 \\
Specific & .251 & .161 & .221 & .194 \\
\hline
\end{tabular}

Note $-W P=$ whole passage $;=$ with pauses. 
pause was included between sentences during RSVP. In addition, general questions were more accurately answered than specific ones $[\mathrm{F}(1,46)=77.75$, MSe $=$ $.015]$. In subsequent analyses comparing these subjects to the control subjects who answered the questions without reading the passages, it was found that the question type effect was stronger among the subjects who had read the passages. This was indicated by an interaction between reading group and question type that was reliable in an analysis involving performance in the whole-passage condition $[\mathrm{F}(2,69)=3.90$, MSe $=$ $.010]$ and in an analysis involving performance in the RSVP condition $[\mathrm{F}(2,69)=4.62, \mathrm{MSe}=.009]$.

The results of Experiment 4 were quite consistent with the prediction that introducing pauses between sentences of an RSVP passage would reduce the discrepancy between question answering performance associated with skimming and rapid sequential reading. Although the critical interaction was reliable only by a one-tailed test, it is supported by converging evidence from Experiment 1, in which reliable differences between skimming and RSVP were found, and from earlier studies (Chen, 1982; Juola et al., 1982; Potter et al., 1980) that included intersentence pauses and failed to find any advantage for skimming over RSVP.

It is conceivable that subjects made use of these pauses to concentrate processing on segments of text (e.g., individual words or coherent propositions) that were included in a limited-capacity working memory buffer and that this processing produced more accurate comprehension and more reliable encoding of information into long-term memory. When pauses did not occur between sentences, performance on both general and specific information suffered relative to performance in the skimming condition. This pattern of results seriously questions the validity of practice effects as an explanation of the differences between skimming and rapid sequential reading.

The major reason that the disadvantage of rapid sequential reading was apparent for both types of questions might be that subjects in Experiment 4 were less adept than those in Experiment 1 at skimming for gist-relevant as opposed to detailed information. (This might also help explain the overall poorer performance in Experiment 4.) Evidence for this is based on the observation that the question type effect for skimming in Experiment 1 was twice as large as the effect obtained in Experiment 4. In addition, when the correlations between how far into a passage one must read to answer a question and probability of correctly answering the question were calculated for the skimming condition in Experiment 4, the correlation among general questions was not reliable and the correlation among specific questions $(r=-.35$ for both rates of skimming) was much less than in Experiment 1. Thus, there seems to have been less of a tendency for subjects in Experiment 4 to curtail processing of detailed information once the first part of a passage had been read. There was also a reliable correlation between location and probability of correct answer for general questions under RSVP with intersentence pauses $(r=-.37)$. The pattern of correct responses tabulated according to which half of a passage contained the answer confirmed this pattern of correlations, as in Experiment 1. This outcome points up a general disadvantage of using skimming as a baseline against which RSVP performance is to be compared. Variations in skimming strategies or ability can influence in an arbitrary fashion the types of questions that will exhibit deficits.

\section{EXPERIMENT 5}

The intersentence pauses used during rapid sequential reading in Experiment 4 may have had their primary effect on encoding of information into memory rather than on the process of comprehension itself. On the other hand, if buffering segments of text information in working memory is a reasonable means of achieving comprehension during rapid sequential reading, then it should be possible to show improvements in comprehension during RSVP by promoting the process of buffering. To this end, the task of answering predesignated questions that was introduced in Experiment 3 was used in Experiment 5 as a measure of comprehension success. Rather than comparing performance under RSVP to performance in a skimming condition again, attention was focused on two versions of rapid sequential reading. In one case, consecutive sentences were presented without pauses, and in the other case, a pause was inserted between sentences. Individual words, however, were always seen for $100 \mathrm{msec}$ each. The presence of intersentence pauses should afford subjects the opportunity to process passage segments that have been buffered in working memory, enabling them to more accurately integrate propositions and make inferences required to answer the comprehension questions.

\section{Method}

Subjects. Thirty-two subjects were recruited as in the earlier experiments. One subject whose performance in the task was particularly poor was replaced.

Materials and Procedure. The passages and questions from the earlier experiments were used, but this time subjects read all 40 passages under conditions of RSVP, with each word presented for a duration of $100 \mathrm{msec}$. Twenty of the passages were presented with no pauses between sentences, and 20 were presented with intersentence pauses. The order of the two types of RSVP was counterbalanced across subjects. Half of the subjects were given a pause of $500 \mathrm{msec}$ between sentences, and half were given a pause of $1,000 \mathrm{msec}$. All other aspects of the experiment were the same as in Experiment 3, except that the type of video monitor used and centering of words were as described in Experiment 4.

\section{Results and Discussion}

Question answering was evaluated as in the earlier experiments, and the mean proportion correct in each condition is shown in Table 3. An analysis of variance 
Table 3

Mean Proportion of Questions Answered in Experiment 5

\begin{tabular}{ccccc} 
& \multicolumn{4}{c}{ Pause Duration } \\
\cline { 2 - 5 } $\begin{array}{c}\text { Question } \\
\text { Level }\end{array}$ & \multicolumn{2}{c}{$500 \mathrm{Msec}$} & \multicolumn{2}{c}{$1,000 \mathrm{Msec}$} \\
\cline { 2 - 5 } & RSVP & RSVP+P & RSVP & RSVP+P \\
\hline General & .508 & .606 & .586 & .703 \\
Specific & .500 & .547 & .512 & .633 \\
\hline
\end{tabular}

Note-P= with pause.

of these data indicated that performance was reliably better when a pause was given between sentences $[\mathrm{F}(1,30)=5.42, \mathrm{MSe}=.054]$, even though words were seen for the same amount of time in both conditions. The only other effect that approached significance was the main effect of question type $[F(1,30)=3.40$, $\mathrm{MSe}=.026, \mathrm{p}<.08]$, suggesting that general questions tended to be answered more accurately than specific questions. The size of the question type effect, however, was no greater than that obtained by control subjects who attempted to answer the questions without having read the passages.

As expected, passage comprehension during rapid sequential reading was significantly improved when brief pauses were inserted between sentences. This was true even though the words themselves were viewed for the same amount of time in all conditions. It was hypothesized that these pauses would provide subjects a chance to concentrate on processing information that had accumulated in working memory and that this processing would enhance comprehension. The enhancement of comprehension was apparent for gist-relevant as well as detailed information, implying that conceptual processing activities during RSVP ranging from macrostructure formation to comprehension of individual propositions may be aided by accumulation of concepts in a limitedcapacity working memory buffer.

Although length of the intersentence pauses had no reliable effect, there was a tendency for longer pauses to produce better performance. One reason that the 1,000-msec pause might not have provided much extra benefit is that both pause durations were longer than the typical end-of-sentence reading time effects observed in other paradigms (e.g., Aaronson \& Scarborough, 1977; Just \& Carpenter, 1980; Mitchell \& Green, 1978). The critical stages of end-of-sentence integration processes may not require longer than $500 \mathrm{msec}$.

\section{GENERAL DISCUSSION}

Evident from experiments on eye movements during reading and the capabilities of the oculomotor system suggest that readers make fixations that average about $200-250 \mathrm{msec}$ because of two types of constraint: (1) time required to plan and initiate the next eye movement and (2) time required for conceptual processing of information that is fixated (e.g., Rayner \& Pollatsek, 1981; Salthouse et al., 1981). If both of these hypothesized constraints exist, attempts to improve reading efficiency by eliminating the need to make eye movements through the use of rapid sequential reading (e.g., Juola et al., 1982; Juola et al., Note 3) will solve only half the problem. Reducing the duration for which each segment of text is exposed during RSVP below typical fixation durations (e.g., $200 \mathrm{msec}$ ) should interfere with conceptual processing if the second constraint applies.

Indirect evidence for the validity of the conceptual processing constraint has been provided by Just and Carpenter (1980), who have shown that time spent viewing a word is related to that word's conceptual characteristics. They assumed that conceptual processing of a word occurs primarily while the reader views the word. If viewing time is prematurely terminated, conceptual processing should be incomplete and comprehension should suffer (cf. Just et al., 1982). This hypothesis was tested in a series of experiments involving RSVP of individual words for as little as $86 \mathrm{msec}$ each.

The ability to answer questions about and to summarize the content of short passages was significantly reduced, relative to performance when skimming, when subjects attempted to read at comparable rates under RSVP. Rapid sequential reading in these cases involved presentation durations as little as half that of an average eye fixation. Moreover, subjects experienced difficulty finding the answers to predesignated questions during rapid sequential reading, but they performed very well while skimming. These results imply that both comprehension and long-term memory encoding problems arise when readers are allowed only brief glimpses of words.

The successful performance of subjects in RSVP experiments involving presentation of single sentences (Fischler \& Bloom, 1980; Juola et al., 1982) or series of pictures (Intraub, 1981; Potter, 1976) probably was due to the fact that only a relatively low level of comprehension was required to detect a single target concept or to prime lexical decisions. Comprehension of multiproposition facts, however, involves a more complex integration of prior knowledge and new information that appears to take more time than is available during brief glimpses of a passage's words.

The claim that text processing deficits associated with RSVP were due to conceptual processing limitations was reinforced by two further experiments in which memory and comprehension performance under RSVP was enhanced by inserting pauses between sentences. The pauses produced only minor changes in the perceptual characteristics of RSVP. But they were expected to allow subjects to more readily process information that had accumulated in working memory (e.g., reconstruct propositions from key words that had 
been identified) due to postponement of processing caused by abbreviated viewing of each word.

It is reasonable to assume, therefore, that one manner in which readers can cope with rapid sequential reading is to buffer limited amounts of information until an opportunity for processing is made available. Interrupting the RSVP sequence more frequently (e.g., at the end of each clause) should place a smaller burden on the capacity of the buffer and could produce even better comprehension (cf. Aaronson \& Scarborough, 1977; Jarvella, 1979).

Alternatively, rapid sequential presentation of text could be arranged to avoid presenting information for durations that are less than the average for normal eye fixations. Juola et al. (1982) have shown that when windows averaging 15 character spaces are shown for $200 \mathrm{msec}$, question answering performance is as good as that achieved through skimming. This technique should produce comprehension and memory results that parallel skimming performance, since it approximates the pattern of visual information seen by skimmers. Although skimmers skip a significant portion of the text (Just et al., Note 1), it is highly likely that windows of 15 characters and more are not always seen clearly enough to allow semantic processing of all the words in a window, so subjects likely miss a good deal of the information in this case as well. The critical aspect of this version of RSVP is the fact that subjects are allowed amounts of time to process each window that are similar to the duration of eye fixations during reading.

The success of these two approaches to enhancing comprehension during rapid sequential reading carries two important implications for the relationship between eye fixation durations and conceptual processing during reading. First, it is apparent that the general length of fixation durations is related to conceptual processes that occur during comprehension. Abbreviating these durations produces serious comprehension and memory problems that cannot be accounted for by the concomitant changes in perceptual processing. Second, the time course of conceptual processing of words seems to be more flexible than previously believed. When there is time enough for only minimal conceptual processing of each word as it appears, readers are able to postpone more complex operations, probably by buffering early data such as lexical information at least until a sentence boundary is reached.

A less dramatic example of this flexibility was apparent in Juola et al.'s (1982) simulation of skimming through presentation of windows of text. Although the windows varied in the complexity of their constituent words, each was presented for the same duration. Subjects apparently were able to distribute their conceptual processing in a manner that compensated for unduly brief visual presentation of more complex words. These results. combined with the possibility of postponing some conceptual processing (as implied by the effectiveness of intersentence pauses), suggest that conceptual processing of a word can be spread over more than just the next one or two words in the text. This "smearing" of conceptual processing may well be responsible for a good deal of the unaccounted for variance in regression analyses of free viewing times.

\section{REFERENCE NOTES}

1. Just, M. A., Carpenter, P. A., \& Masson, M. E. J. What eye fixations tell us about speed-reading, skimming, and normal reading. Manuscript in preparation, 1983.

2. Masson, M. E. J., Carpenter, P. A., \& Just, M. A. Comprehension of gist and details by speed-readers, skimmers, and normal readers. Manuscript in preparation, 1983.

3. Juola, J. F., Cocklin, T., Chen, H. -C., \& Granaas, M. Effects of segment size and structure on reading RSVP text. Paper presented at the annual meeting of the Psychonomic Society, Minneapolis, Minnesota, November 1982.

\section{REFERENCES}

Aaronson, D., \& Scarborough, H. S. Performance theories for sentence coding: Some quantitative models. Journal of Verbal Learning and Verbal Behavior, 1977, 16, 277-303.

Arnold, D. C., \& Tinker, M. A. The fixational pause of the eyes. Journal of Experimental Psychology, 1939, 25, 271-280.

Bartlett, F. C. Remembering: $A$ study in experimental and social psychology. Cambridge, England: Cambridge University Press, 1932.

Bransford, J. D., \& Johnson, M. K. Contextual prerequisites for understanding: Some investigations of comprehension and recall. Journal of Verbal Learning and Verbal Behavior, 1972, 11, 717-726.

ChEN, H. -C. Comprehension and memory in reading rapid serial presentations of text. Unpublished doctoral dissertation, University of Kansas, 1982.

Dooling, D. J., \& Lachman, R. Effects of comprehension on retention of prose. Journal of Experimental Psychology, 1971, 88, 216-222.

Fischler, I., \& Bloom, P. A. Rapid processing of the meaning of sentences. Memory \& Cognition, 1980, 8, 216-225.

GiBson, E. J., \& LEVIN, H. The psychology of reading. Cambridge: M.I.T. Press, 1975.

Green, D. W., Mitchell, D. C., \& Hammond, E. J. The scheduling of text integration processes in reading. Quarterly Journal of Experimental Psychology, 1981, 33A, 455-464.

IntrauB, H. Rapid conceptual identification of sequentially presented pictures. Journal of Experimental Psychology: Human Perception and Performance, 1981, 7, 604-610.

JARVELLA, R. J. Immediate memory and discourse processing. In G. H. Bower (Ed.), The psychology of learning and motivation (Vol. 13). New York: Academic Press, 1979.

Juola, J. F., Ward, N., \& McNamara, T. Visual search and reading rapid, serial presentations of letter strings, words, and text. Journal of Experimental Psychology: General, 1982, 111, 208-227.

JUSt, M. A., \& CARPEnter, P. A. A theory of reading: From eye fixations to comprehension. Psychological Review, 1980, 87, 329-354.

Just, M. A., Carpenter, P. A., \& Woolley, J. D. Paradigms and processes in reading comprehension. Journal of Experimental Psychology: General, 1982, 111, 228-238.

KIERAS, D. E. Initial mention as a signal to thematic content in technical passages. Memory \& Cognition, 1980, 8, 345-353.

KIERAS, D. E. The role of major referents and sentence topics in the construction of passage macrostructure. Discourse Processes, 1981, 4, 1-15. 
Kintsch, W., \& van DiJk, T. A. Toward a model of text comprehension and production. Psychological Review, 1978, 85, 363-394.

Masson, M. E. J. Cognitive processes in skimming stories. Journal of Experimental Psychology: Learning, Memory, and Cognition, 1982, 8, 400-417.

Millen, J. R., \& Kintsch, W. Readability and recall of short prose passages: A theoretical analysis. Journal of Experimental Psychology: Human Learning and Memory, 1980, 6, 335-354.

Mitchell, D. C., \& Green, D. W. The effects of context and content on immediate processing in reading. Quarterly Journal of Experimental Psychology, 1978, 30, 609-636.

ORTONY, A. Remembering, understanding, and representation. Cognitive Science, 1978, 2, 53-69.

Perfetti, C. A., \& Lesgold, A. M. Discourse comprehension and sources of individual differences. In M. A. Just \& P. A. Carpenter (Eds.), Cognitive processes in comprehension. Hillsdale, N.J: Erlbaum, 1977.

Potter, M. C. Short-term conceptual memory for pictures. Journal of Experimental Psychology: Human Learning and Memory, 1976, 2, 509-522.

Potter, M. C., Kroll, J. F., \& Harris, C. Comprehension and memory in rapid sequential reading. In $R$. S. Nickerson (Ed.), Attention and performance VIII. Hillsdale, N.J: Erlbaum, 1980.

Poulton, E. C. Time for reading and memory. British Journal of Psychology, 1958, 49, 230-245.

Poulton, E. C. Rapid reading. Journal of Documentation, 1963, 19, 168-172.

RAYNER, $K$. The perceptual span and peripheral cues in reading. Cognitive Psychology, 1975, 7, 65-81.

RAYNER, K. Eye movements in reading and information processing. Psychological Bulletin, 1978, 85, 618-660.

Rayner, K., Inhoff, A. W., Morrison, R. E., Slowiaczek, M. L., \& Bertera, J. H. Masking of foveal and parafoveal vision during eye fixations in reading. Journal of Experimental
Psychology: Human Perception and Performance, 1981, 7, 167-179.

Rayner, K., \& McConkie, G. W. What guides a reader's eye movements? Vision Research, 1976, 16, 829-837.

RAynen, K., \& McConkie, G. W. Perceptual processes in reading: The perceptual spans. In A. S. Reber \& D. L. Scarborough (Eds.), Toward a psychology of reading. Hillsdale, N.J: Erlbaum, 1977.

Rayner, K., \& Pollatsek, A. Eye movement control during reading: Evidence for direct control. Quarterly Journal of Experimental Psychology, 1981, 33A, 351-373.

REED, A. V. Microcomputer display timing: Problems and solutions. Behavior Research Methods \& Instrumentation, 1979, 11, 572-576.

Salthouse, T. A., \& Ellis, C. L. Determinants of eye-fixation duration. American Journal of Psychology, 1980, 93, 207-234.

Salthouse, T. A., Ellis, C. L., Diener, D. C., \& Somberg, B. L. Stimulus processing during eye fixations. Journal of Experimental Psychology: Human Perception and Performance, 1981, 7,611-623.

STICHT, T. G. Comprehending reading at work. In M. A. Just \& P. A. Carpenter (Eds.), Cognitive processes in comprehension. Hillsdale, N.J: Erlbaum, 1977.

TAYLOR, S. E. An evaluation of forty-one trainees who had recently completed the "Reading Dynamics" program. In E. P. Bliesmer \& R. C. Staiger (Eds.), Eleventh yearbook of the National Reading Conference. Milwaukee: National Reading Conference, 1962.

Thibadeau, R., Just, M. A., \& Carpenter, P. A. A model of the time course and content of reading. Cognitive Science, in press.

(Received for publication August 25, 1982; revision accepted December 23, 1982.) 